The University of San Francisco

USF Scholarship: a digital repository @ Gleeson Library |

Geschke Center

Business Analytics and Information Systems

School of Management

$3-2014$

\title{
Engineering Enterprises for Emergent Change
}

Steven Alter

University of San Francisco, alter@usfca.edu

Follow this and additional works at: http://repository.usfca.edu/at

Part of the Business Commons

\section{Recommended Citation}

Alter, S. 2014. "Engineering Enterprises for Emergent Change," Working Paper, University of San Francisco, 2014.

This Article is brought to you for free and open access by the School of Management at USF Scholarship: a digital repository @ Gleeson Library | Geschke Center. It has been accepted for inclusion in Business Analytics and Information Systems by an authorized administrator of USF Scholarship: a digital repository@ Gleeson Library | Geschke Center. For more information, please contact repository@usfca.edu. 


\title{
Engineering Enterprises for Emergent Change
}

\author{
Steven Alter \\ School of Management \\ University of San Francisco \\ San Francisco, USA \\ alter@usfca.edu
}

\begin{abstract}
This paper uses work system theory (WST) and two of its extensions to provide an integrated perspective on engineering enterprises for emergent change. This paper starts by explaining six basic assumptions and distinctions related to emergent change. It introduces four frameworks or models related to WST including the work system framework, work system life cycle model, a theory of workarounds, and a work system metamodel. It shows how each framework or model can help in identifying different aspects of engineering for emergent change and also can be the basis of guidelines for that purpose. Overall, this paper provides a unique way to think about the engineering of enterprises. In addition, it explains a combination of concepts and frameworks that provide a path toward engineering for emergent change.
\end{abstract}

Keywords- enterprise engineering, enterprise transformation, emergent change, work system, work system framework, workaround

\section{A NEW PERSPECTIVE ON ENGINEERING ENTERPRISES FOR EMERGENT CHANGE}

This paper is a contribution to the 8th TEE Workshop on Transformation \& Engineering of Enterprises. It uses work system theory and several of its extensions to explain a unique perspective on the transformation and engineering of enterprises. Instead of assuming that the transformation and engineering of enterprises is basically about imagining and specifying how enterprises will operate at a point in the future, this paper assumes that the transformation and engineering of enterprises occurs through a combination of planned and unplanned change. With that assumption, enterprise engineering broadens to a combination of engineering for planned change and engineering for unplanned change that may or may not be consistent with whatever was planned by managers and designers.

The idea of engineering enterprises for emergent change might seem like a bit of an oxymoron because the general concept of engineering brings connotations of producing detailed specifications of an end product that satisfies clear requirements. This paper's basic premise is that emergent change probably will occur, and therefore that the engineering of enterprises should try to channel inevitable emergent change in beneficial directions. That approach is quite different from the "design in advance" assumptions underlying BPM software, enterprise architecture, and other similar approaches for influencing enterprise transformation through rigorously documented specifications of enterprise structure and operation in the future.
This paper's contribution is in two areas. First, it illustrates what engineering for emergence might mean, thereby providing a unique way to think about the transformation and engineering of enterprises. Second, it explains a specific combination of concepts and frameworks that provide a path toward engineering for emergent change.

This paper starts by explaining basic assumptions and distinctions related to emergent change. Next it uses WST and several of its extensions as an integrated perspective on emergent change. That perspective provides a way to visualize the work system components in which change can occur, the nature of planned and unplanned change, the microdynamics of unplanned change, and a broader view of how emergent change leads to learning. The starting point for this perspective is the work system framework, which identifies nine elements of a basic understanding of a work system. The work system life cycle model provides a big picture view of how a work system changes over time through a combination of planned and unplanned change. The theory of workarounds describes the rationale through which workarounds and other emergent changes occur within work systems. The work system metamodel reveals more of the microdynamics of emergent change. In combination, these ideas provide background leading to the identification of many aspects of engineering for emergent change.

\section{BASIC ASSUMPTIONS ABOUT EMERGENT CHANGE}

This paper builds upon six basic assumptions about the nature of emergent change.

Emergent properties vs. emergent change. There is a fundamental difference between the emergent properties of systems in organizations and emergent change that occurs within such systems. Emergent properties of systems are system properties that are more than properties of the individual components. For example, the scalability, reliability, and adaptability of a system are related to a combination of different components such as processes, participants, and technologies. In contrast, emergent change [1] is unplanned change that is improvised or designed in work situations when work system participants discover reasons to change existing methods, procedures, and relationships. Emergent change within a work system may change any particular component or any combination of components. Thus, emergent change is qualitatively different from top-down planned change that tends to occur through projects to which management allocated resources. 


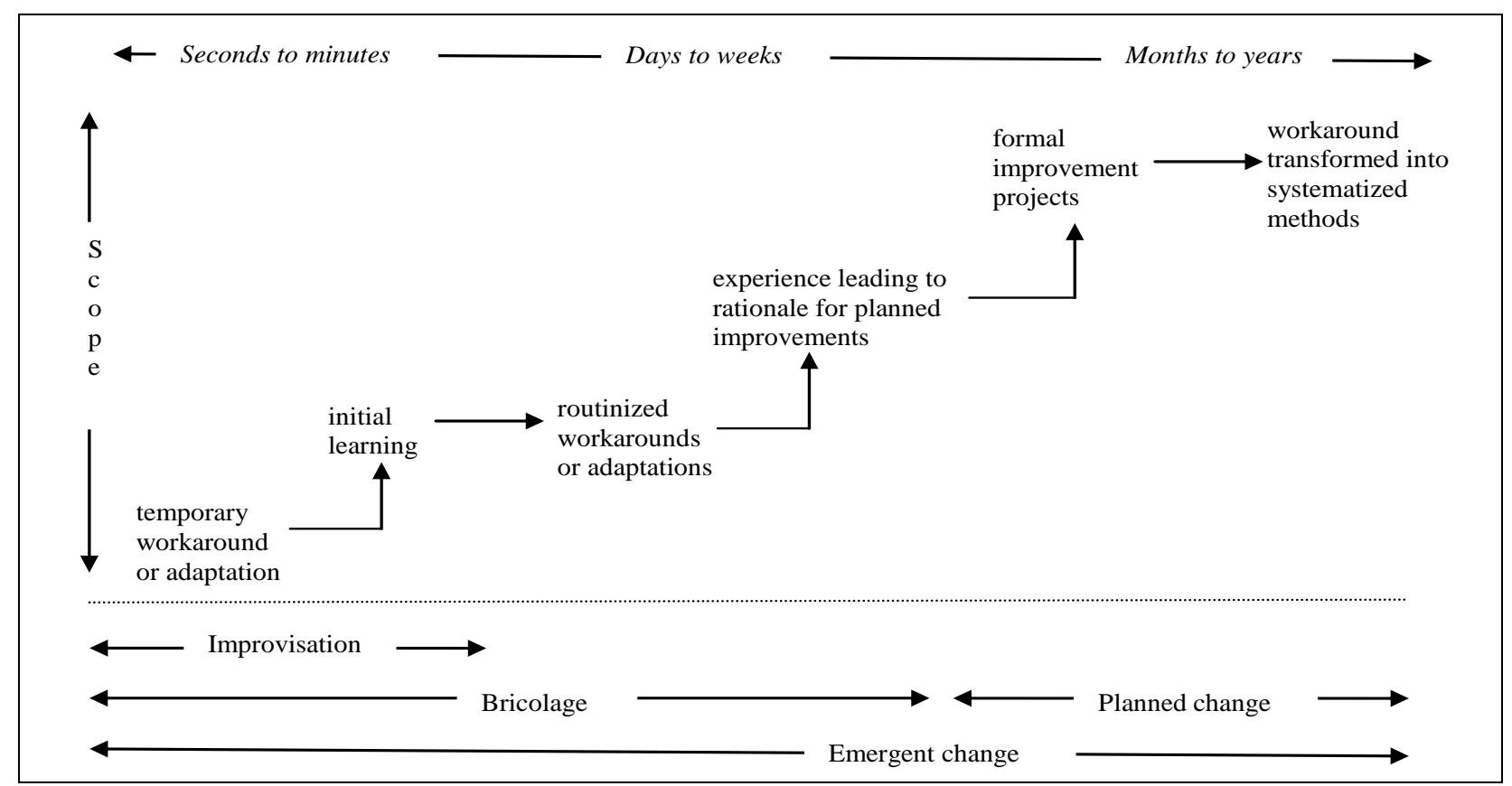

Fig. 1. From temporary workarounds to emergent change [2]

Human agency. Emergent change is largely the product of human agency even though it is possible to imagine some cases in which emergent change in systems and enterprises occurs as a result of interactions between seemingly independent adaptations. Accidents and happenstance may create new options or foreclose others, but the options still must be selected by people.

Beneficial and/or harmful changes. Emergent changes may be beneficial in some respects and harmful and others, as when a nursing group's procedural change to make it easier to serve their patients may have the negative consequence of generating inaccurate data for the hospital's medical record system. Thus, there should be no assumption that any particular emergent change is necessarily beneficial for an enterprise.

From improvisations to learning and adaptation. Figure 1 illustrates how emergent change can grow out of small improvisations and workarounds that occur in a time frame of seconds-to-minutes [2]. Transient or persistent obstacles lead to a temporary workaround or adaptation, which leads to learning about its effectiveness, which in turn may lead to a routinized workaround or adaptation. Additional learning from experience with those incremental changes provides a rationale for planned improvements, some of which may require formal projects that result in formally systematized methods.

Loose vs. tight specifications. In many situations there are choices between specifying processes in detail versus specifying processes somewhat loosely and giving people considerable freedom to perform their work activities in ways that seem right to them. Tightly defined structures and controls tend to constrain emergent change. Loosely defined structures and controls tend to permit or even encourage change. One of the sociotechnical principles described by $[3,4]$ was "minimum critical specification," i.e., using tight specifications only where tightness is genuinely beneficial. By that principle, process designers should specify only what is essential and should leave the rest to the judgment of the people doing the work. Similarly, some consultants and theorists have called for simultaneously tight and loose management (e.g., $[5,6])$ that provides clear guidelines but does not create unnecessary constraints.

Emergent change in work systems. It is relatively easy to visualize emergent change occurring within a particular work system within an enterprise. It is much more difficult to imagine how simultaneous emergent change processes in different parts of an enterprise can redirect the course of an entire enterprise. For that practical reason, this paper will focus on emergent change at the work system level rather than at the level of entire enterprises or large organizations. In the conclusions, this paper will return to entire enterprises or large organizations to show how the ideas related to work systems are also related emergent change on a larger scale.

\section{WORK SYSTEMS AS THE LOCUS OF EMERGENT CHANGE}

This paper's perspective on engineering enterprises for emergent change assumes that most emergent change occurs at the work system level rather than the enterprise level. The following sections explain how basic ideas encapsulated in work system theory and two of its extensions provide a basis for engineering for emergent change.

Definition of work system. A work system is a system in which human participants and/or machines perform processes and activities using information, technology, and other resources to produce products/services for internal or external customers. Enterprises that grow beyond a largely improvised start-up phase can be viewed as consisting of multiple work systems. Typical business enterprises contain work systems that procure materials from suppliers, produce products, deliver products, find customers, create financial reports, hire 
employees, coordinate work across departments, and perform many other functions. There are a number of important special cases of work systems. Information systems are work systems all of whose activities are devoted to processing information. Projects are work systems designed to produce specific products/ services and then go out of existence. Sociotechnical work systems have human participants, in contrast with totally automated work systems which operate autonomously and automatically after being launched.

Work system theory. As explained in depth in [7], work system theory (WST) is a perspective for thinking about systems in organizations in which the unit of analysis is the work system. WST defines the term work system and includes two central frameworks that provide a static view of a work system as it exists during a particular time interval and a dynamic view of how a work system changes over time. The two frameworks are called the work system framework (static view) and the work system life cycle model (dynamic view). [7] identifies a number of extensions of WST that build upon the WST core to address other issues. Two of those extensions that are directly relevant to engineering for emergent change are a theory of workarounds and a work system metamodel that re-interprets concepts in the work system framework in a way that is useful for detailed analysis of a work system.

The following four sections introduce ways in which WST and two of its extensions are directly relevant for understanding emergent change. Each section summarizes a framework or model related to WST and then summarizes ways in which that framework or model is directly relevant for understanding emergent change. In turn, the sections focus on the work system framework, work system life cycle model, theory of workarounds, and a recent version of the work system metamodel. Subsequent sections go a step further by showing how ideas related to each framework or model are relevant to engineering for emergent change.

\section{WORK SYSTEM FRAMEWORK}

The work system framework (Figure 2) represents a work system in terms of nine elements in a basic understanding of the work system's form, function, and environment during a period when it is relatively stable, even though incremental changes may occur during that period. As shown in Figure 2, processes and activities, participants, information, and technologies are viewed as completely within the work system; customers and products/services may be partially inside and partially outside because customers often participate in the processes and activities within the work system and because products/services take shape within the work system; environment, infrastructure, and strategies are viewed as outside the work system even though they have direct effects within the work system.

Relevance to understanding emergent change. Each element of the work system framework is a possible area for emergent change. The following list (based on a similar list in [2]) shows how emergent change can appear in changes in each element:

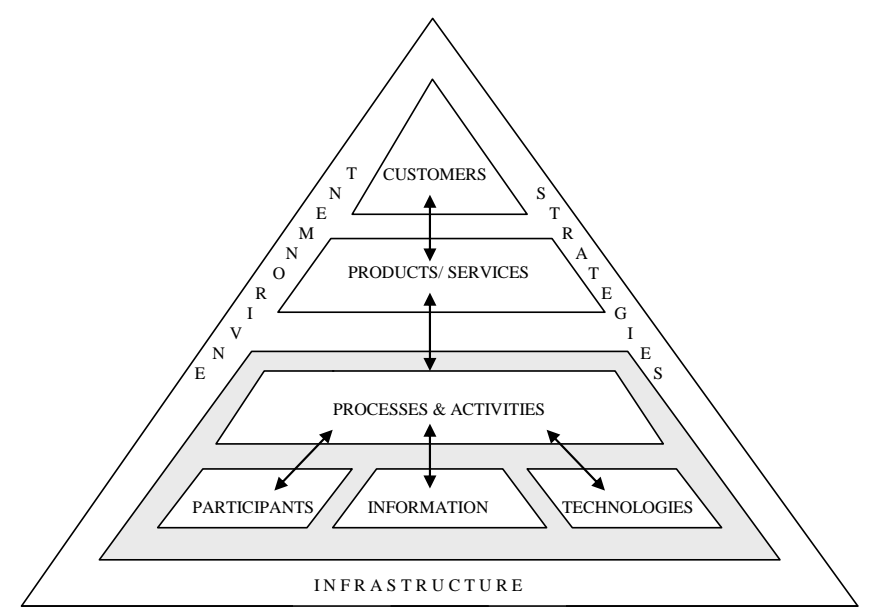

Fig. 2. Work system framework [7]

- Processes and activities. Do the work in a different way (e.g., skip steps, add steps, change the sequence of steps, use different techniques for performing steps), possibly because of the cumbersome nature of prescribed processes and activities.

- Participants. Allow or assign different participants to do the work (e.g., people who are not fully trained, are overqualified, or are temporary workers), possibly because the people who should do the work are unavailable.

- Information. Do the work with different information (e.g., proceed with partial information or use information from a different source such as a local shadow system), possibly because of problems with the quality, timeliness, completeness, or cost of the prescribed information.

- Technologies. Work around bugs and/or inadequate features (e.g., use old technology instead of prescribed technology, use prescribed technology in a non-prescribed manner, create spreadsheets or other personal information systems to bypass or augment sanctioned information systems, or bypass the technology altogether).

- Products/services. Produce physical or informational products/services that deviate from previous expectations or specifications from the work system's customers, designers, or management. (The term products/services recognizes that outputs of most work systems combine product-like and service-like characteristics.)

- Customers. Produce products/services for previously unserved internal or external customers. Alternatively, withhold products/services from some of the work system's previous customers to minimize problems for the work system or its participants or customers.

- Environment. Perform work differently in response to situations in the surrounding environment, such as demand changes, challenges from organizational culture, or competitive issues, regulations, or business policies.

- Infrastructure. Bypass expected uses of infrastructure (technical, informational, and human resources shared with other work systems), e.g., by accessing shared information 
in a different way. Alternatively, use infrastructural resources, such as human infrastructure, to overcome shortcomings in the work system's core elements.

- Strategies. Work around existing strategies of the work system, department, or enterprise if those strategies or their misalignments pose obstacles to achieving goals.

\section{WORK SYSTEM LIFE CYCLE MODEL}

The work system life cycle model (Figure 3) represents the iterative process through which work systems evolve over time via a combination of planned change (projects involving allocation of resources by management) and emergent (unplanned) change that occurs locally, often with no management involvement or even awareness, through adaptations, bricolage [8,9] and workarounds. Planned change occurs through projects that include initiation, development, and implementation phases. Development involves creation or acquisition of resources including hardware, software, documentation, and training materials that are required for implementation of desired changes in the organization.

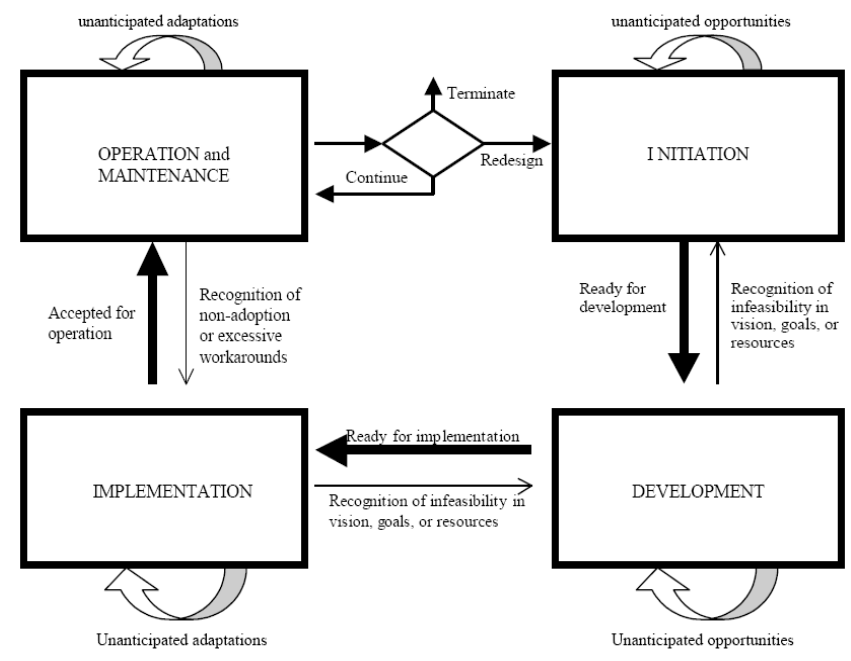

Fig. 3. Work system life cycle model [7]

The WSLC represents emergent change using inwardfacing arrows representing ongoing adaptations, bricolage, and workarounds that change aspects of the current work system without separate allocation of significant project resources. With its iterative nature and focus on work systems rather than software per se, the WSLC is fundamentally different from the SDLC, Rational Unified Process (RUP) and other IT-oriented process models that are designed to provide guidance for executing software development projects.

Relevance to understanding emergent change. All of the inward-facing arrows in the WSLC represent points where emergent change occurs. In the operation and maintenance phase, the emergent change occurs when workarounds, adaptations, and local experimentation lead to new practices in the operational work system.

- Transient workarounds. Work system participants are confronted with obstacles and need to do something to work around those obstacles. Sometimes the obstacles are exception conditions that have been noted previously, and may even be mentioned in formal procedures for handling exceptions. In other cases the obstacles are unanticipated, and still need to be dealt with to produce expected results. Thus, workarounds are unplanned changes because they are deviations from the expected process. (The next section looks at workarounds more depth.)

- Repeated workarounds and adaptations. Work system participants discover that prescribed processes and resources at hand are insufficient for working efficiently and effectively. They change processes accordingly, often through local adjustments that managers do not perceive.

- Bug fixes. In some cases, unplanned changes are related to fixing bugs or improving awkward software features that can be fixed without a formal project.

Emergent change also occurs during the other phases. Emergent change during the initiation phase occurs when the preliminary analysis during that phase discovers issues that affect the initially assumed project scope. Emergent change occurs during development when the team discovers opportunities to work more efficiently and/or problems that require changes in methods and project scope. Emergent change during implementation occurs when the implementation process itself reveals opportunities to use resources more effectively and/or problems that require changes in software or other outputs of the development phase.

\section{THEORY OF WORKAROUNDS}

The previous section noted that emergent change in operational work systems often stems from workarounds, adaptations, and local experimentation lead to new practices. The WSLC identifies where emergent changes occur, but it does not explain mechanisms through which they happen. That is the goal of the theory of workarounds illustrated in Figure 4.

This theory covers most types of workarounds in 300+ examples in the literature related to operational systems (as explained in [2]). It is based on a broad definition of workaround that clarifies the preconditions for the occurrence of a workaround and encompasses most other definitions of workaround in the literature. A workaround is a goal-driven adaptation, improvisation, or other change to one or more aspects of an existing work system in order to overcome, bypass, or minimize the impact of obstacles, exceptions, anomalies, mishaps, established practices, management expectations, or structural constraints that are perceived as preventing that work system or its participants from achieving a desired level of efficiency, effectiveness, or other organizational or personal goals.

Italicized terms on the left side of Figure 4 identify generic steps for perceiving the need for a workaround and then creating it. system participants create workarounds by identifying obstacles and deciding what to do. The theory combines ideas from the theory of planned behavior [10], agency theory [11], and concepts related to improvisation and bricolage $[8,9,12,13,14]$. 


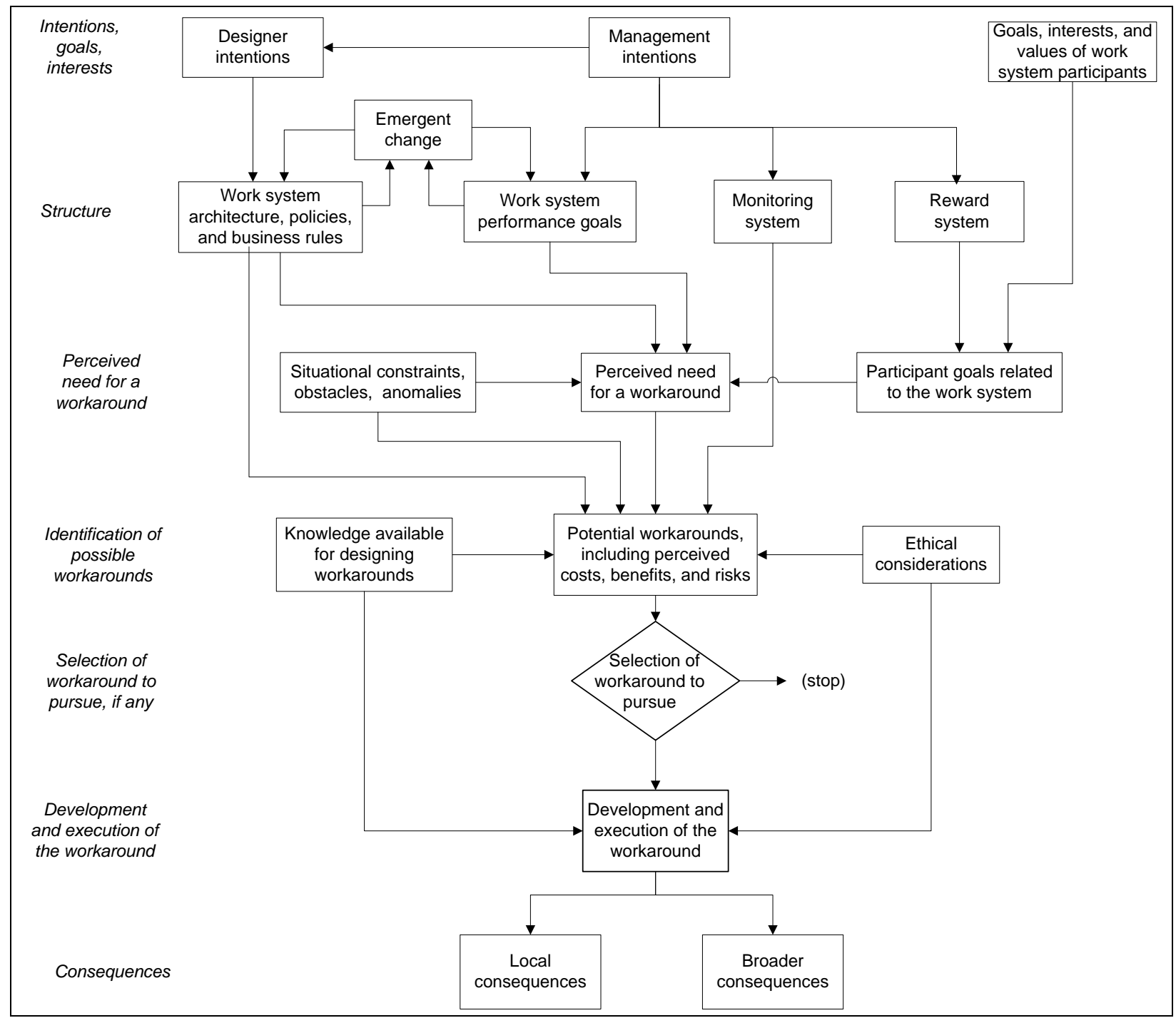

Fig. 4. Theory of Workarounds [2]

Relevance to understanding emergent change. Workarounds are a major source of emergent change. Each step in Fig. 4 will be discussed briefly, with the term emergent change sometimes used instead of workaround because the current topic is emergent change that often comes from the adoption and repeated use of workarounds. The first two steps reflect the context within which emergent change occurs. Specific emergent changes are developed and executed through a typical problem solving process that includes perceiving the need for a change, identifying possible changes, selecting a change to pursue (if any), developing and executing the change, and finally, reaping the consequences.

- Intentions, goals, and interests of management, designers, and work system participants form the context for emergent change, which appears below the layer for intentions because aspects of systems may have emerged over time through adaptations and past workarounds that occurred without management guidance or formal projects.
- $\quad$ Structure includes the architecture and characteristics of the work system, work system performance goals, the monitoring system, and the reward system. The loop related to emergent change for the work system says that management and designer intentions affect architecture, policies, business rules, and performance goals, all of which may be factors in emergent change. Completing the loop, emergent change affects the work system's structure.

- Perceived need for a workaround is based on a combination of the work system's architecture and performance goals, situational constraints, obstacles, and anomalies, and work system - related goals of participants.

- Identification of possible workarounds (i.e., possible directions for emergent change) is triggered by the perceived need for workarounds. Consideration of costs, benefits, and risks starts with perceived obstacles and the perceived need for changes. Design-related knowledge is essential for considering any workaround seriously. 
Perceived benefits, costs, and risks for each approach that is considered reflect the effort of eliminating obstacles and longer term consequences of the approach taken. Monitoring systems affect the likelihood of detection for potentially questionable changes. Ethical considerations may also come into play in some cases.

- Selection of workaround to pursue, if any, reflects concepts from the theory of planned behavior [10], such as attitudes toward the behavior, subjective norms, and perceived behavioral controls, plus concepts from agency theory such as moral hazard and information asymmetry.

- Development and execution of the workaround can occur in minutes in simple cases where process steps are bypassed or modified slightly, or can take weeks or months if software must be designed and implemented.

- Local consequences and broader consequences complete the picture. With transient workarounds, the main advantages involve eliminating temporary obstacles. The emergent changes that stems from such workarounds may involve improved workflows or other improvements. The local disadvantages may include failure of the workaround or creation of other problems, such as distorting information used later in the same work system.

\section{WORK SYSTEM METAMODEL}

Figure 5 is a recent version of a work system metamodel that augments the work system framework. The work system framework helps in summarizing a work system and achieving mutual understanding of the scope and nature of a work system. It is less effective as a tool for detailed analysis. The more complete and rigorous metamodel is more precise about concepts required to support deeper analysis without requiring terminology (e.g., objects and classes) that is impenetrable to most business professionals.

The metamodel makes concepts in the work system framework clearer, more rigorous, and more useful for work system documentation and software development. This creates a bridge between summary level descriptions and more detailed models and subsystems during analysis and design. It does that without requiring the precision, terminology, and notation of BPMN or of rigorous software specifications. When used with a second layer that identifies common characteristics, metrics, and principles for specific elements, it can support traceability between a summary level analysis and more detailed analysis and documentation by IT specialists.

The metamodel is a more detailed re-interpretation of the elements of the work system framework. Information becomes informational entity, technology is divided into tools and automated agents, activities are performed by three types of actors, and so on. Representation decisions in the metamodel try to maximize understandability while revealing potential omissions from an analysis or design process.

Fig. 5 hides a large number of important attributes such as characteristics, metrics, and principles that apply to specific elements and relationships in the metamodel. Analysts using the metamodel would consider and apply the hidden attributes while defining the problem or opportunity, evaluating the "as is" work system, and justifying proposed improvements that would appear in the "to be" work system.

Relevance to understanding emergent change. The metamodel reveals more about where emergent change occurs. A work system contains activities that use resources to produce products/services. Those products/services may become resources for other activities and/or may be received and used by the work system's customers.

Emergent change occurs when work system participants perform activities using resources that are different from those that used in established practices and methods. The various types of informational, technological, human, and other resources in Fig. 5 are all possible locations for change. For example, emergent change might involve substitution of certain participants for other participants, use of different information of various types, use of different technologies, and changes in other types of resources.

\section{IMPLICATIONS}

This paper proposes the possibility of engineering enterprises for emergent change. The previous five sections provide frameworks, models, and related diagrams that can be used to look at emergent change from different perspectives and at different levels of detail. This section applies those ideas in several ways. First it identifies general implications of those frameworks, models, and diagrams under the assumption that engineering for emergent change might be a good idea. Next it uses each of this paper's five diagrams to identify specific topics and concerns that should be included in an attempt to engineer for emergent change. Later, the paper's conclusion returns to the question of whether the whole idea of engineering enterprises for emergent change is potentially beneficial and whether it contradicts taken-for-granted assumptions about the nature of engineering in general and about the nature of enterprise engineering.

\section{A. Implications of the five diagrams from previous sections}

The previous five sections provide frameworks, models, and diagrams that can be used to look at emergent change from different perspectives and at different levels of detail. This section shows that the diagram in each of the previous five sections has direct implications for any attempt to engineer for emergent change.

The diagram describing the path from temporary workarounds to emergent change (Fig. 1) shows that transient workarounds can lead to learning, which in turn leads to incremental improvements in routines that may become systematized methods instituted through formal projects.

- $\quad$ Engineering for emergent change should enable and encourage initial workarounds and their routinization, but only where the workarounds and the new routines will be beneficial. Thus, engineering for emergent change should encompass two sometimes contradictory ideas, flexibility and control. 


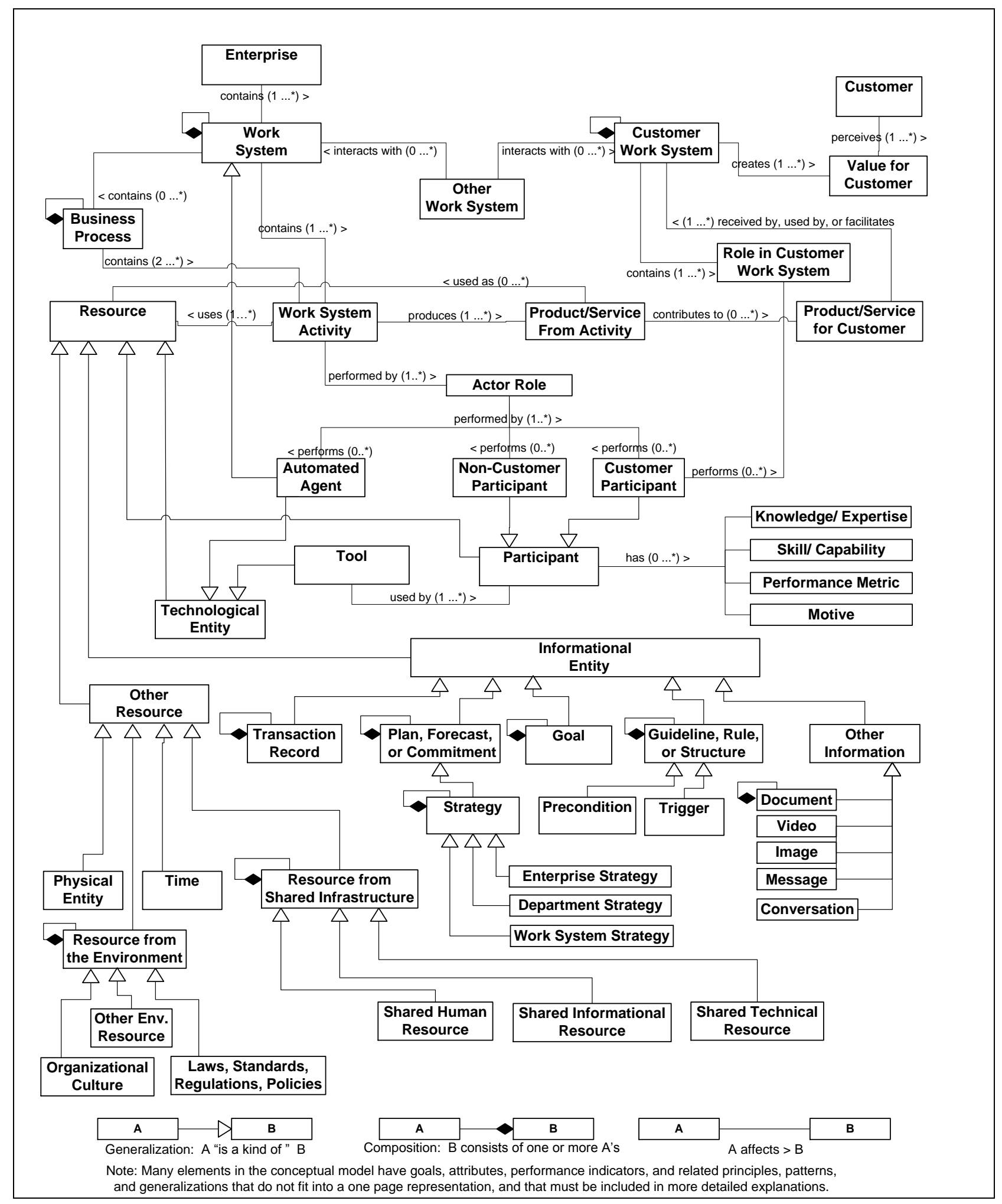

Fig. 5. Metamodel for integrated analysis and design of sociotechnical and technical systems (revision of metamodel in [15])

The work system framework (Fig. 2) provides a high-level view of the elements of a basic understanding of how a particular work system operates within its own context.

- All of the elements of a basic understanding of the work system are relevant to engineering for emergent change.
Ignoring any particular element could result in missed opportunities or unnecessary difficulties.

The WSLC (Fig. 3) provides a high-level view of how work systems change over time. The inwardly facing arrows in 
the four phases of the WSLC indicate that emergent change can occur in each phase.

- Engineering for emergent change should pay attention to the possibility of emergent change in any of the four phases. The operation and maintenance phase should assume that a work system currently in operation is not static and unchanging, and that its management should try to attain a beneficial balance of flexibility and control. The three project phases should recognize that initial management intentions might be overridden by emergent changes during the project.

The theory of workarounds (Fig. 4) summarizes a rationale for imagining and deploying workarounds, which are often a starting point for emergent change. The rationale includes steps in creating workarounds and factors related to each step.

- Each step and factor in Fig. 4 is relevant to whether workarounds will be created, whether they will be beneficial initially, and whether they will become part of organizational routines. Engineering for emergent change should maximize the likelihood that beneficial workarounds will be created and that inappropriate workarounds will not be attempted.

The work system metamodel (Fig. 5) shows that every activity in a work system uses certain resources and produces certain products/services that may become resources for other activities in the work system and/or may be received and used by the work system's customers.

- Changes in any entity type or relationship in Fig. 5 might be an enabler or consequence of emergent change. Engineering for emergent change should consider emergent change possibilities for each entity type or relationship with a potential for significant emergent changes.

\section{B. Potential guidelines for engineering for emergent change}

Assume that an enterprise's executives, managers, and technical experts want to support emergent change. Listed below are a number of guidelines that could lead in that direction. The list does not try to evaluate whether possible changes are a good idea. The concluding section will explain that designing for emergent change could be a very good idea in some situations and a very bad idea in other situations. The purpose of the guidelines is to illuminate possibilities.

Guidelines based on Fig. 1, the path from an initial workarounds to routinization. Fig. 1 represents the "happy path" by which initial workarounds lead to favorable experience with beneficial changes in organization routines, which in turn leads to improvements in formally authorized systems. Related engineering guidelines include the following:

- Facilitate the creation of initial workarounds where necessary.

- Monitor the effectiveness of workarounds and their positive and negative impacts elsewhere.

- Provide feedback about that effectiveness.
- Encourage routinization of workarounds that are effective and do not cause significant problems.

Guidelines based on Fig. 2, the work system framework. It is possible to propose guidelines for each of the nine elements of the work system framework and for the work system as a whole. The following list is illustrative. Other guidelines might be added.

- Work system as a whole. 1) Engineer sociotechnical work systems consistent with the sociotechnical principle of minimum critical specification (mentioned previously). 2) Engineer to accommodate the appropriate level of scalability, resilience, adaptability, and other important characteristics of the work system as a whole. 3) Engineer in conjunction with monitoring systems and reward systems that encourage beneficial adaptations and discourage harmful adaptations.

- Processes and activities. Engineer for visibility of the operation of processes and activities along with related performance metrics and feedback that could support emergent change.

- Participants. Engineer to enhance visibility of whether work system participants are fully capable of identifying needs for workarounds or adaptations, imagining appropriate workarounds, and taking appropriate action.

- Information. Engineer to facilitate availability of information for performing workarounds, for providing feedback, for supporting other control activities, and for learning.

- Technologies. Engineer to achieve appropriate technical flexibility and adaptability.

- Products/services. Engineer to facilitate changes that might enable use of appropriately modified methods to produce somewhat different products/services if demand or other relevant conditions change.

- Customers. Engineer to include feedback about customer participation (related to co-production opportunities), customer use of the products/services (where possible), and customer satisfaction.

- Environment. Engineer to include environmental scanning that identifies inconsistencies or conflicts between work system operation and relevant factors in the immediate environment.

- Infrastruture. Engineer for appropriate use of shared human, informational, and technical infrastructure and to expand or contract the use of that infrastructure as situations change.

- Strategies. Engineer for consistency with current enterprise or department strategies, and for adaptability if strategies change in foreseeable directions.

Guidelines based on Fig. 3, the work system life cycle model. The operation and management phase was addressed by guidelines related to the work system framework, Fig. 2. 
The other three phases of the WSLC (initiation, development, and implementation) are all subprojects within a larger planned change project. Engineering for emergent change is relevant for the three project phases only in a limited sense since those phases are terminate after producing their deliverables.

- Operation and management. Since this is the operation of the work system, the guidelines related to the work system framework (Fig. 2) are applicable.

- Initiation. Engineer the initiation phase to make it easy to question whether the initially intended scope of the project is actually the correct scope and to adapt the project's scope accordingly.

- Development. Identify possibilities or needs to change the scope or details of the project as unforeseen issues and possibilities are explored and become understood.

- Implementation. Identify possibilities or needs to change the details of the project as issues and possibilities become evident during implementation.

Guidelines based on Fig. 4, the theory of workarounds. Each step in the theory of workarounds brings opportunities or reminders related to engineering for emergent change.

- Intention, goals, interests. Try to separate designer and management intentions from the details of the work system. Awareness of that distinction may provide guidance in developing workarounds where necessary.

- Structure. Recognize that emergent change may have modified whatever were the initial work system architecture, policies, and business rules. Recognize that structure includes not only the work system's structure but also the monitoring system, reward system, and performance goals for the work system.

- Perceived need for a workaround. Facilitate recognition of needs for workarounds by helping work system participants visualize how situational constraints, obstacles, and anomalies affect their ability to attain the work system's performance goals and their own personal goals related to the work system.

- Identification of possible workarounds. Support the effort to identify workaround possibilities. Do this by supporting the previous step, by making relevant knowledge available, and by identifying impacts on others as a reminder of possible ethical issues.

- Selection of workaround. Support evaluation of alternative workarounds by identifying possible consequences.

- Development and execution of workaround. Provide flexibility that makes it relatively convenient to develop and execute appropriate workarounds.

- Consequences. Have a tracking system that recognizes workarounds and collects information related to their effectiveness.

Guidelines based on Fig. 5, the work system metamodel Since most of the entity types in the metamodel are directly related to elements in the work system framework (Fig. 2), the guidelines mentioned previously in relation to the work system framework are generally relevant to those entity types. For example, the guideline related to information mentioned for Fig. 2 is relevant to most of the different types of informational entity types in Fig. 5. Additional guidelines related to the nature of specific entity types in the metamodel (e.g., transaction records vs. plans vs. goals vs. conversations) will not be mentioned here because that would extend this paper's length. Suffice it to say that it would be possible to provide many additional guidelines related to the specific entity types and relationships in the metamodel.

\section{CONCLUSION: IS ENGINEERING FOR EMERGENT CHANGE A GOOD IDEA?}

This paper's goal was to explain how WST and two of its extensions provide an integrated perspective on engineering for emergent change. The paper was organized around five diagrams, with each successive diagram leading to additional implications and guidelines related to the possibility of engineering for emergent change at the level of work systems.

The feasibility of engineering at that level does not imply that engineering entire enterprises for emergent change is either possible or beneficial. This conclusion identifies related issues.

\section{A. Is engineering for emergent change possible at the work system level?}

The foregoing sections illustrate that the combination of WST and two of its extensions provides a conceptual basis for engineering work systems for emergent change. Focusing on work systems provides a unit of analysis within which emergent change occurs (Fig. 1). Emergent changes may be related to any of the nine elements of the work system framework (Fig. 2). Emergent changes can occur during any of the four phases of the WSLC (Fig. 3). At least part of the microdynamics of emergent change is described by the steps and factors in the theory of workarounds (Fig. 4). The specifics of an emergent change can be viewed in a more detailed way by looking at the entity types and relationships in the metamodel (Fig. 5). Implications and guidelines related to engineering for emergent change were mentioned for each step in that sequence.

The above concepts and rationale for engineering for emergent change in work systems does not demonstrate the practicality of performing that type of engineering. A convincing empirical demonstration would require a lengthy, multi-site research project that traced projects in great detail. That type of demonstration is far beyond the current scope because this paper's more limited goal is to explain a new perspective on engineering for emergent change.

The empirical evidence is not available, but it is possible to explain how engineering for emergent change could be included in the analysis and design process for improving a work system. Assume that a sociotechnical work system is to be improved through a combination of process improvement, better information, better technology, and better skills and knowledge for system participants. A typical analysis and 
design process would produce a tentative description of how the proposed, improved work system should operate.

Engineering for emergent change would examine the proposed work system in depth and would try to identify specific areas where workarounds and adaptations seem most likely to occur in the future. That exercise could not possibly anticipate all future conditions and all future personal motives of work system participants and stakeholders. Therefore it could not anticipate all possible emergent changes. On the other hand, it could use design patterns for workarounds and adaptations that could be developed based on existing research such as the many situations compiled to justify the theory of workarounds in [2]. For example, if the work system called for logging in before performing intermittent transactions, it would be possible to ask whether multiple participants in a group would save time by using a single login for hours at a time, thereby confusing any statistics about who was responsible for which transaction. If the work system included use of complex software such as ERP, it would be possible to ask whether that software might permit work system participants to store some data in fields intended for other purposes or whether it would permit them to bypass certain data entry expectations that they would view as too cumbersome. If the process called for inconvenient signoffs, as sometimes happens in hospitals and clinics, it would be possible to ask whether the work system participants would be likely to use workarounds such as bypassing the signoff step and filling in the details many hours later. If the software addressed only part of specific informational needs, it would be more likely that work system participants would develop and use personal spreadsheets and shadow systems that would help them do their work despite perceived shortcomings of the official software.

The exercise of engineering work systems for emergent change would identify areas where workarounds and adaptations that launch emergent change seem most likely. For each of those situations the designers and relevant stakeholders would ask whether the workaround would be acceptable and under what circumstances. At minimum, the implementation of the new version of the work system could include explanations and documentation about why certain workarounds would be acceptable and other workarounds would be harmful or dangerous. With more effort, monitoring systems could be augmented to record evidence that workarounds were occurring and to provide that evidence to managers who would have to take whatever corrective action, if any, seemed appropriate.

Parts of the above process occur in practice today, even though most systems analysis and design textbooks, analysis and design research, and BPM research pay little attention to workarounds and adaptations. The contribution of the current research is to show how a work system perspective might provide organized support for engineering for emergent change in work systems. That support might occur in the form of implications and guidelines such as those mentioned in this paper, and perhaps might even include compilations of common types of workarounds and related design patterns.

\section{B. Is engineering for emergent change desirable at the work system level?}

Even though engineering for emergent change at the work system level seems possible, it is reasonable to ask whether ir is desirable. Executives and managers of some enterprises might want to engage in it, but executives and managers of many other enterprises might want to define and enforce topdown definitions of processes. With that stance, they would want to treat process definitions as rules rather than guidelines and would try to prevent emergent change.

Regardless of what executives and managers might want, however, assuming that emergent change will not occur is simply denying reality in many situations. It is likely that emergent change will occur in an enterprise's sociotechnical work systems unless that enterprise is willing to invest in extensive monitoring systems or highly automated processes. That assumption is supported by empirical research. For example, recognized experts on organizational routines have shown that organizational routines can generate endogenous change as a result of simply carrying out the routine [16]. More recently, a study of invoice processing in four Norwegian organizations [17] "generated hundreds of unique patterns that changed significantly during a five- month period without any apparent external intervention. Changes did not appear to reflect improved performance or learning." [17] also found that increased automation can increase variation under some circumstances.

The fact that emergent change occurs in many situations implies that the choice about how to engineer a sociotechnical work system boils down to a choice about what to consider in the engineering process. Incorporating the high likelihood that emergent change will occur requires the additional effort of trying to anticipate endogenous changes and deciding what to do about them. For any particular change possibility, it might be more appropriate to do nothing, to explain the pros and cons to work system participants before they attempt workarounds, to set up monitoring systems for identifying when changes occur, if ever, or to try to create software capabilities that prevent or guide emergent changes. An alternative approach is to ignore emergent change when engineering work systems, thereby treating emergent change as someone else's problem that will be faced after the project is over. A possible rationale for that approach is the assumption that anticipating most emergent changes is too difficult and that the responses should be left to the local managers after the changes start to occur. In either case, having a clear stance about how to deal with emergent change during the engineering process clarifies goals and expectations related to everyday reality.

\section{Is enterprise engineering for emergent change either possible or desirable?}

This paper noted earlier that it would focus on emergent change at the work system level rather than at the level of entire enterprises. Engineering for emergent change at the enterprise level presents a far more difficult problem. The discussions surrounding the five diagrams showed that it is comparatively easy to visualize emergent change occurring 
within a particular work system within an enterprise. It is much more difficult to imagine how simultaneous emergent change processes in different parts of an enterprise can redirect the course of an entire enterprise in a coherent way.

The most familiar examples of technology-related emergent change that cross entire organizations involve the introduction of new technologies. Decades ago early types of spreadsheet software were sometimes introduced to large organizations by providing enthusiasts with those capabilities and then publicizing and building on initial applications that proved useful. That approach was possible because the technology was introduced without making it an essential component of mandatory or mission critical business processes. More recently, similar approaches have been used to introduce social networking capabilities into organizations. (e.g., [18]).

While those approaches proved successful, seeding technology in an organization and seeing what happens does not sound like "enterprise engineering." The whole notion of engineering involves careful specification of requirements and internally consistent specifications and documentation of features and capabilities. Enterprise engineering for emergent change would start with a requirement and would create specifications and documentation that cross the enterprise.

Given the scale of that challenge and the widely recognized difficulties of implementing enterprise-wide software that has already been developed, focusing on the work system level seems the most practical approach for making progress in the direction of enterprise engineering for emergent change. Starting at a work system level would involve introducing concepts and tools related to emergent change into analysis and design processes used when trying to improve important work systems. If those approaches and tools proved useful, a number of important work systems would have been engineered with some emphasis on emergent change. Whether that would add up to "enterprise engineering for emergent change" would be a matter of opinion. At minimum, it would be a step in that direction.

\section{What comes next?}

The name of the TEE series of workshops, "Transformation \& Engineering of Enterprises," presents significant challenges even if one assumes that an engineered enterprise will hold steady for a while, an assumption that is overwhelmingly contradicted by the recent history of business enterprises. Based on that history, engineering for emergent change seems an essential part of any realistic engineering of enterprises.

This paper presented a specific combination of concepts and frameworks that provide a path toward engineering enterprises for emergence. Along the way, it illustrated what engineering for emergence might mean, thereby providing a unique way to think about the transformation and engineering of enterprises. This paper focused on the work system level because that seemed an appropriate level for making progress, especially since enterprises can be viewed as a sum of their work systems (plus whatever emerges from the interactions of those work systems). The ideas make sense, but the practicality of applying those ideas has not been demonstrated empirically.

The next step is to develop and try out a set of tools and methods that can be used in conjunction with existing analysis and design methods. Those tools and methods would achieve initial success if they help system designers and other stakeholders identify possible workarounds and adaptations and decide what to do about those possibilities. Follow-on research would identify workarounds that actually occurred after the proposed work systems were implemented. It would observe the extent to which the anticipated workarounds actually occurred and the extent to which the discussion of workarounds helped work system participants make good decisions about whatever workarounds they actually attempted.

\section{REFERENCES}

[1] W. J. Orlikowski, "Improvising organizational transformation over time: A situated change perspective. Information systems research," (7)1, 1996, pp. 63-92.

[2] S. Alter, "Theory of Workarounds," Communications of the Association of Information Systems, 34(55), 2014, pp. 1041-1066.

[3] A. Cherns, "Principles of socio-technical design," Human Relations, (2) 9,1976 , pp. 783-792

[4] A. Cherns, "Principles of sociotechnical design revisted", Human Relations, 40(3),1987 pp. 153-161.

[5] T. Peters, and R. Waterman, R., In search of excellence: Lessons from America's best-run corporations. New York: Warner, 1982.

[6] K. A. Merchant, "The control function of management," Sloan Management Review, (23)4, 1982, pp. 43-55.

[7] S. Alter, "Work System Theory: Overview of Core Concepts, Extensions, and Challenges for the Future," Journal of the Association for Information Systems, 14(2), 2013, .pp. 72-121.

[8] C. Ciborra, "Notes on Improvisation and Time in Organizations," Accounting, Management and Information Technologies, (9)2, 1999, pp. 77-94.

[9] C. Ciborra, The Labyrinths of Information: Challenging the Wisdom of Systems, Oxford, UK: Oxford University Press, 2002.

[10] I. Ajzen, I., "The theory of planned behavior", Organizational Behavior and Human Decision Processes, (50), 1991, pp. 179-211.

[11] M. K. Eisenhardt, "Agency theory: An assessment and review", Academy of Management Review, (14), 1989, pp. 57-74.

[12] C. Levi-Strauss, The Savage Mind, Chicago: University of Chicago Press, 1967.

[13] K. E. Weick, "The collapse of sensemaking in organizations: the Mann Gulch disaster", Administrative Science Quarterly, (38), 1993, pp. 628652.

[14] T. Baker and R. E. Nelson, "Creating Something from Nothing: Resource Construction through Entrepreneurial Bricolage", Administrative Science Quarterly, (50), 2005, pp. 329-366.

[15] S. Alter, "From Resources and Activities to Value for Customers within Systems of Service Systems," Proceedings of SIG-SVC 2013 Workshop, Dec. 15, 2013, Milan Italy

[16] Feldman, M. and B.T. Pentland, "Reconceptualizing Organizational Routines as a Source of Flexibility and Change," Administrative Science Quarterly, (48):1, 2003. pp. 94-118.

[17] B. T. Pentland, T. Haerem, D. Hillson "The (N)Ever-Changing World: Stability and Change in Organizational Routines," Organization Science, 22(6), 2011, pp. 1369-1383.

[18] Riemer, K., \& Scifleet, P. (2012, January). Enterprise social networking in knowledge-intensive work practices: A case study in a professional service firm. ACIS 2012: Proceedings of the 23rd Australasian Conference on Information Systems 2012 (pp. 1-12). 
